\title{
Generalized Exponential Trichotomies for Abstract Evolution Operators on the Real Line
}

\author{
Nicolae Lupa ${ }^{1}$ and Mihail Megan ${ }^{2,3}$ \\ ${ }^{1}$ Faculty of Economics and Business Administration, West University of Timişoara, Boulevard Pestalozzi 16, \\ 300115 Timişoara, Romania \\ ${ }^{2}$ Academy of Romanian Scientists, Independenţei 54, 050094 Bucharest, Romania \\ ${ }^{3}$ Faculty of Mathematics and Computer Science, West University of Timişoara, Boulevard V. Pârvan 4, \\ 300223 Timişoara, Romania \\ Correspondence should be addressed to Mihail Megan; megan@math.uvt.ro
}

Received 22 May 2013; Accepted 30 August 2013

Academic Editor: Messaoud Bounkhel

Copyright (C) 2013 N. Lupa and M. Megan. This is an open access article distributed under the Creative Commons Attribution License, which permits unrestricted use, distribution, and reproduction in any medium, provided the original work is properly cited.

This paper considers two trichotomy concepts in the context of abstract evolution operators. The first one extends the notion of exponential trichotomy in the sense of Elaydi-Hajek for differential equations to abstract evolution operators, and it is a natural extension of the generalized exponential dichotomy considered in the paper by Jiang (2006). The second concept is dual in a certain sense to the first one. We prove that these types of exponential trichotomy imply the existence of generalized exponential dichotomy on both half-lines. We emphasize that we do not assume the invertibility of the evolution operators on the whole space $X$ (unlike the case of evolution operators generated by differential equations).

\section{Introduction}

The notion of exponential dichotomy plays a central role in the qualitative theory of differential equations, dynamical systems, and many other domains (see, e.g., [1-4] and the references therein). While dichotomy assumes the existence of two complementary projections, trichotomy implies the existence of three projections. The exponential trichotomies are split into two qualitative different classes, depending on the behavior of the evolution operators with respect to the structural projections. The first type, introduced by Sacker and Sell [5], involves a continuous decomposition of the state space into three closed subspaces (the stable subspace, the unstable subspace, and the neutral subspace) (for more details about this type of trichotomy we refer the reader to [6-10]). The second one, introduced by Elaydi and Hajek in [11], implies the existence of exponential dichotomy on both half lines with structural projections $P_{+}$and $P_{-}$such that $P_{+} P_{-}=P_{-} P_{+}=P_{-}$. As far as we know, the case of exponential trichotomy in the sense of Elaydi-Hajek has been studied before only for reversible evolution operators in $[12,13]$, and in particular for differential equations in $[11,14,15]$.

This paper considers two trichotomy concepts in the sense of Elaydi-Hajek in the general case of abstract evolution operators. The first one extends the exponential trichotomy in [11] to evolution operators which are not invertible on the whole space $X$, and it is a natural extension of the generalized exponential dichotomy considered in [1]. This type of exponential dichotomy was defined by Muldowney in [4] for differential equations without assuming conditions (7) and (8). The second concept is dual in a certain sense to the first one and implies the existence of generalized exponential dichotomy on both $\mathbb{R}_{-}$and $\mathbb{R}_{+}$, with projection valued functions $P_{-}$and $P_{+}$, respectively, such that

$$
P_{+}(t) P_{-}(t)=P_{-}(t) P_{+}(t)=P_{+}(t), \quad \forall t \in \mathbb{R} .
$$

The aim of the paper is to extend some results from [11] to the concepts of exponential trichotomy mentioned above. We note that we do not need to assume neither the invertibility of 
the evolution operators on the whole space $X$ nor a bounded growth condition on $U(t, s)$.

\section{Preliminary Notions}

Let $X$ be a real or complex Banach space and let $\mathscr{B}(X)$ be the Banach algebra of all bounded linear operators on $X$. The norms on $X$ and on $\mathscr{B}(X)$ will be denoted by $\|\cdot\|$. Also, we consider $\Delta=\left\{(t, s) \in \mathbb{R}^{2}: t \geq s\right\}$ and the subsets

$$
\Delta_{-}=\{(t, s) \in \Delta: t \leq 0\}, \quad \Delta_{+}=\{(t, s) \in \Delta: s \geq 0\} .
$$

In this section, we give some preliminary definitions.

Definition 1. An operator valued function $U: \Delta \rightarrow \mathscr{B}(X)$ is said to be an evolution operator (on $X$ ) if the following conditions are satisfied:

$$
\begin{aligned}
& \left.\left(e_{1}\right) U(t, t)=I \text { (the identity operator on } X\right), \text { for } t \in \mathbb{R} ; \\
& \left(e_{2}\right) U(t, s) U\left(s, t_{0}\right)=U\left(t, t_{0}\right), \text { for all } t \geq s \geq t_{0} .
\end{aligned}
$$

If $U(t, s)$ are defined for all $t, s \in \mathbb{R}$ and relation $\left(e_{2}\right)$ holds for all $t, s, t_{0} \in \mathbb{R}$, then we say that $U$ is a reversible evolution operator.

The notion of evolution operator arises naturally from the theory of well-posed evolution equations. Roughly speaking, when the Cauchy problem

$$
\left\{\begin{array}{l}
\dot{u}(t)=A(t) u(t), \quad t \geq s, \\
u(s)=x
\end{array}\right.
$$

is well-posed with regularity subspaces $Y_{t}, t \in \mathbb{R}$, the operator

$$
U(t, s) x:=u(t ; s, x) \quad \text { for } t \geq s, x \in Y_{s},
$$

where $u(\cdot ; s, x)$ is the unique solution of (3), can be extended by continuity to an evolution operator. For more details on well-posed nonautonomous Cauchy problems, we refer the reader to Nagel and Nickel [16] and the references therein.

Definition 2. An operator valued function $P: \mathbb{R} \rightarrow \mathscr{B}(X)$ is said to be a projection valued function if

$$
P^{2}(t)=P(t), \quad \text { for every } t \in \mathbb{R} \text {. }
$$

Definition 3. Three projection valued functions $P_{i}: \mathbb{R} \rightarrow$ $\mathscr{B}(X), i \in\{1,2,3\}$, are called supplementary if

(1) $P_{1}(t)+P_{2}(t)+P_{3}(t)=I$, for $t \in \mathbb{R}$;

(2) $P_{i}(t) P_{j}(t)=0$, for $t \in \mathbb{R}$ and $i, j \in\{1,2,3\}, i \neq j$.

Definition 4. Given an evolution operator $U: \Delta \rightarrow \mathscr{B}(X)$, we say that a projection valued function $P: \mathbb{R} \rightarrow \mathscr{B}(X)$ is invariant for $U$ if

$$
P(t) U(t, s)=U(t, s) P(s), \quad \forall(t, s) \in \Delta .
$$

This implies that the family $\mathscr{R}(P(t)), t \in \mathbb{R}$, of ranges of the projections $P(t)$, is invariant in the sense that if $x \in \mathscr{R}(P(s))$ for some $s \in \mathbb{R}$, then $U(t, s) x \in \mathscr{R}(P(t))$ for all $t \geq s$. For an evolution operator $U$ and a projection valued function $P$ invariant for $U$ such that the restriction of the operator $U(t, s)$ on $\mathscr{R}(P(s))$ viewed as a map from $\mathscr{R}(P(s))$ into $\mathscr{R}(P(t))$, is an isomorphism for certain $(t, s) \in \Delta$, we denote by $U_{P}(s, t)$ the inverse operator from the range of $P(t)$ onto the range of $P(s)$.

Definition 5. Let $U: \Delta \rightarrow \mathscr{B}(X)$ be an evolution operator and let $P: \mathbb{R} \rightarrow \mathscr{B}(X)$ be a projection valued function which is invariant for $U$. We say that $P$ is

(i) compatible with $U$ if the restriction of $U(t, s)$ on $\mathscr{R}(P(s))$ into $\mathscr{R}(P(t))$ is an isomorphism for all $(t, s) \in$ $\triangle$;

(ii) compatible on the left with $U$ if the restriction of $U(t, s)$ on $\mathscr{R}(P(s))$ into $\mathscr{R}(P(t))$ is an isomorphism for all $(t, s) \in \Delta_{-}$;

(iii) compatible on the right with $U$ if the restriction of $U(t, s)$ on $\mathscr{R}(P(s))$ into $\mathscr{R}(P(t))$ is an isomorphism for all $(t, s) \in \Delta_{+}$.

We denote by $\mathcal{U}$ the set of all bounded and continuous functions $u: \mathbb{R} \rightarrow(0, \infty)$ satisfying

$$
\begin{gathered}
\int_{s}^{t} u(\tau) d \tau \longrightarrow \infty \quad \text { as } t \longrightarrow \infty \text { for fixed } s \in \mathbb{R}, \\
\int_{s}^{t} u(\tau) d \tau \longrightarrow \infty \quad \text { as } s \longrightarrow-\infty \text { for fixed } t \in \mathbb{R} .
\end{gathered}
$$

If there is a constant $a>0$ such that $u(t) \geq a$ for all $t \in \mathbb{R}$, then $u \in \mathcal{U}$. In particular, all the positive constants belong to the set $\mathcal{U}$. However, there exist functions different from those mentioned above which belong to $\mathcal{U}$. For example,

$$
u(t)=\frac{1}{1+|t|}, \quad t \in \mathbb{R}
$$

(there exists no $a>0$ such that $u(t) \geq a$ for all $t \in \mathbb{R}$ ).

\section{Generalized Exponential Trichotomies}

In this section, we consider two trichotomy concepts in the sense of Elaydi-Hajek in the general case of abstract evolution operators.

3.1. Generalized $\ell$-Exponential Trichotomy. The notion of exponential trichotomy given below extends the exponential trichotomy in [11] to evolution operators which are not invertible on the whole space $X$, and it is a natural extension of the generalized exponential dichotomy considered in [1].

Definition 6. We say that an evolution operator $U$ has a generalized $\ell$-exponential trichotomy if there exist three supplementary projection valued functions $P_{1}, P_{2}, P_{3}$ and there exist a constant $K \geq 1$ and a function $u \in \mathscr{U}$ such that the following properties hold:

$\left(l_{1}\right) P_{1}$ is invariant for $U$;

$\left(l_{2}\right) P_{2}$ is compatible with $U$; 
$\left(l_{3}\right) P_{3}$ is compatible on the left with $U$;

$\left(l_{4}\right)\left\|U(t, s) P_{1}(s)\right\| \leq K e^{-\int_{s}^{t} u(\tau) d \tau}$, for all $(t, s) \in \Delta$;

$\left(l_{5}\right)\left\|U_{P_{2}}(s, t) P_{2}(t)\right\| \leq K e^{-\int_{s}^{t} u(\tau) d \tau}$, for all $(t, s) \in \Delta$;

$\left(l_{6}\right)\left\|U(t, s) P_{3}(s)\right\| \leq K e^{-\int_{s}^{t} u(\tau) d \tau}$, for all $(t, s) \in \Delta_{+}$;

$\left(l_{7}\right)\left\|U_{P_{3}}(s, t) P_{3}(t)\right\| \leq K e^{-\int_{s}^{t} u(\tau) d \tau}$, for all $(t, s) \in \Delta_{-}$.

If there exists a positive constant $a>0$ such that $u(t) \geq a$ for all $t \in \mathbb{R}$, then we say that $U$ has an $\ell$-exponential trichotomy (in the sense of Elaydi-Hajek).

The notion of $\ell$-exponential trichotomy was introduced in [11] for linear differential equations in finite-dimensional spaces and in [13] for reversible evolution operators. In this paper, we do not assume the invertibility of the evolution operator on the whole space $X$. This degree of generalization is motivated by possible applications to partial differential equations, in which the evolution operators are not invertible (see [17] and the references therein). Furthermore, in [11] the authors require the matrix $A(t)$ to be bounded on the real line. In the case of abstract evolution operators, this corresponds to the assumption that the evolution operators are exponentially bounded (i.e., there exist $\omega>0$ and $M \geq 1$ such that $\|U(t, s)\| \leq M e^{\omega(t-s)}$, for all $\left.t \geq s\right)$. Unfortunately, most of the evolution operators do not possess this property (see [18, pp. 12]).

Remark 7. If $U$ has a generalized $\ell$-exponential trichotomy with projection valued functions $P_{1}, P_{2}$, and $P_{3}$, then we have that

$$
\sup _{t \in \mathbb{R}}\left\|P_{i}(t)\right\|<\infty, \quad \text { for } i \in\{1,2,3\}
$$

that is, the projection valued functions $P_{1}, P_{2}$, and $P_{3}$ are uniformly bounded.

Remark 8. If in Definition 6 we consider $P_{3}(t)=0$ for all $t \in \mathbb{R}$, then we obtain the concept of generalized exponential dichotomy (on the real line) (see [1]). This means that if an evolution operator has a generalized exponential dichotomy (on the real line), then it also has a generalized $\ell$-exponential trichotomy.

Remark 9. If $U$ has a generalized $\ell$-exponential trichotomy with projection valued functions $P_{1}, P_{2}$, and $P_{3}$, then conditions (7) and (8) imply that

$$
\begin{gathered}
U(t, s) P_{i}(s) x \longrightarrow 0 \quad \text { as } t \longrightarrow+\infty, i \in\{1,3\} \\
U_{P_{i}}(t, s) P_{i}(s) x \longrightarrow 0 \quad \text { as } t \longrightarrow-\infty, i \in\{2,3\} .
\end{gathered}
$$

If the evolution operator $U$ is generated by a well-possed evolution equation, then the relations above lead to a geometric description of the extended state space $\mathbb{R} \times X$ of (3). More precisely, it splits into three invariant vector bundles (the stable bundle, the unstable bundle, and the center bundle). For more details about the geometric theory of discrete and continuous nonautonomous dynamical systems, we refer the reader to [19] and the references therein.
One can easily give an example of an evolution operator which has a generalized $\ell$-exponential trichotomy and does not have an $\ell$-exponential trichotomy.

Example 10. The evolution operator $U: \Delta \rightarrow \mathscr{B}\left(\mathbb{R}^{3}\right)$ defined by

$$
\begin{aligned}
& U(t, s)\left(x_{1}, x_{2}, x_{3}\right) \\
& \quad=\left(e^{-\int_{s}^{t} u(\tau) d \tau} x_{1}, e^{\int_{s}^{t} u(\tau) d \tau} x_{2}, e^{-\int_{s}^{t} \operatorname{sign}(\tau) u(\tau) d \tau} x_{3}\right)
\end{aligned}
$$

has a generalized $\ell$-exponential trichotomy with canonical projections for each $u \in \mathcal{U}$. However, it does not have an $\ell$-exponential trichotomy for the function $u$ considered in relation (9).

The main goal of the paper is to extend some results from [11] to the general case of abstract evolution operators. We note that we do not need to assume neither the invertibility of the evolution operators on the whole space $X$ nor a bounded growth condition on the evolution operators (unlike the case of differential equations with bounded coefficients as in [11]).

Theorem 11. An evolution operator $U: \Delta \rightarrow \mathscr{B}(X)$ has a generalized $\ell$-exponential trichotomy if and only if there exist two projection valued functions $P_{+}, P_{-}: \mathbb{R} \rightarrow \mathscr{B}(X)$ which are invariant for $U$ and there exist a constant $K \geq 1$ and $a$ function $u \in \mathcal{U}$ such that the following properties hold:

(1) $P_{+}(t) P_{-}(t)=P_{-}(t) P_{+}(t)=P_{-}(t)$, for all $t \in \mathbb{R}$;

(2) $\sup _{t \leq 0}\left\|P_{+}(t)\right\| \leq M_{+}$and $\sup _{t \geq 0}\left\|P_{-}(t)\right\| \leq M_{-}$;

(3) The restriction of $U(t, s)$ on $\mathscr{R}\left(Q_{+}(s)\right)$ into $\mathscr{R}\left(Q_{+}(t)\right)$ is an isomorphism for all $(t, s) \in \Delta_{+}$;

(4) The restriction of $U(t, s)$ on $\mathscr{R}\left(Q_{-}(s)\right)$ into $\mathscr{R}\left(Q_{-}(t)\right)$ is an isomorphism for all $(t, s) \in \Delta_{-}$;

(5) $\left\|U(t, s) P_{+}(s)\right\| \leq K e^{-\int_{s}^{t} u(\tau) d \tau}$, for all $(t, s) \in \Delta_{+}$;

(6) $\left\|U_{Q_{+}}(s, t) Q_{+}(t)\right\| \leq K e^{-\int_{s}^{t} u(\tau) d \tau}$, for all $(t, s) \in \Delta_{+}$;

(7) $\left\|U(t, s) P_{-}(s)\right\| \leq K e^{-\int_{s}^{t} u(\tau) d \tau}$, for all $(t, s) \in \Delta_{-}$;

(8) $\left\|U_{Q_{-}}(s, t) Q_{-}(t)\right\| \leq K e^{-\int_{s}^{t} u(\tau) d \tau}$, for all $(t, s) \in \Delta_{-}$;

where $Q_{+}(t)=I-P_{+}(t)$ and $Q_{-}(t)=I-P_{-}(t), t \in \mathbb{R}$.

Proof. Necessity. We consider

$$
P_{+}(t)=P_{1}(t)+P_{3}(t), \quad P_{-}(t)=P_{1}(t), \quad \text { for } t \in \mathbb{R} \text {. }
$$

It is easy to see that the first two conditions hold and we have that

$$
Q_{+}(t)=P_{2}(t), \quad Q_{-}(t)=P_{2}(t)+P_{3}(t), \quad \forall t \in \mathbb{R} .
$$

These imply that $U(t, s)_{\mid \mathscr{R}\left(Q_{+}(s)\right)}: \mathscr{R}\left(Q_{+}(s)\right) \rightarrow \mathscr{R}\left(Q_{+}(t)\right)$ is an isomorphism for all $(t, s) \in \Delta_{+}$with the inverse

$$
U_{Q_{+}}(s, t)=U_{P_{2}}(s, t),
$$


and $U(t, s)_{\mid \mathscr{R}\left(Q_{-}(s)\right)}: \mathscr{R}_{\left(Q_{-}(s)\right)} \rightarrow \mathscr{R}\left(Q_{-}(t)\right)$ is an isomorphism for all $(t, s) \in \Delta_{-}$with

$$
U_{\mathrm{Q}_{-}}(s, t)=U_{P_{2}}(s, t) P_{2}(t)+U_{P_{3}}(s, t) P_{3}(t) .
$$

Hence,

$$
U_{Q_{-}}(s, t) Q_{-}(t)=U_{P_{2}}(s, t) P_{2}(t)+U_{P_{3}}(s, t) P_{3}(t),
$$

for all $(t, s) \in \Delta_{-}$. We first prove that $U_{Q_{-}}(s, t)$ considered above is correctly defined. Indeed, for every $x \in \mathscr{R}\left(Q_{-}(t)\right)$, we have that

$$
\begin{aligned}
U_{Q_{-}} & (s, t) x \\
= & U_{P_{2}}(s, t) P_{2}(t) x+U_{P_{3}}(s, t) P_{3}(t) x \\
= & P_{2}(s) U_{P_{2}}(s, t) P_{2}(t) x+P_{3}(s) U_{P_{3}}(s, t) P_{3}(t) x \\
= & P_{2}(s)\left(U_{P_{2}}(s, t) P_{2}(t) x+U_{P_{3}}(s, t) P_{3}(t) x\right) \\
& +P_{3}(s)\left(U_{P_{2}}(s, t) P_{2}(t) x+U_{P_{3}}(s, t) P_{3}(t) x\right) \\
= & Q_{-}(s)\left(U_{P_{2}}(s, t) P_{2}(t) x+U_{P_{3}}(s, t) P_{3}(t) x\right)
\end{aligned}
$$

which belongs to $\mathscr{R}\left(Q_{-}(s)\right)$. Moreover, a simple computation shows that

$$
\begin{array}{r}
U(t, s) U_{Q_{-}}(s, t) Q_{-}(t)=Q_{-}(t), \\
U_{Q_{-}}(s, t) U(t, s) Q_{-}(s)=Q_{-}(s), \\
\text { for }(t, s) \in \Delta_{-} .
\end{array}
$$

For $(t, s) \in \Delta_{+}$, we have

$$
\begin{aligned}
\left\|U(t, s) P_{+}(s)\right\| \leq & \left\|U(t, s) P_{1}(s)\right\| \\
& +\left\|U(t, s) P_{3}(s)\right\| \leq 2 K e^{-\int_{s}^{t} u(\tau) d \tau}, \\
\left\|U_{Q_{+}}(s, t) Q_{+}(t)\right\| & =\left\|U_{P_{2}}(s, t) P_{2}(t)\right\| \leq K e^{-\int_{s}^{t} u(\tau) d \tau} .
\end{aligned}
$$

If $(t, s) \in \Delta_{-}$, then we get

$$
\begin{gathered}
\left\|U(t, s) P_{-}(s)\right\|=\left\|U(t, s) P_{1}(s)\right\| \leq K e^{-\int_{s}^{t} u(\tau) d \tau}, \\
\left\|U_{Q_{-}}(s, t) Q_{-}(t)\right\| \leq\left\|U_{P_{2}}(s, t) P_{2}(t)\right\|+\left\|U_{P_{3}}(s, t) P_{3}(t)\right\| \\
\leq 2 K e^{-\int_{s}^{t} u(\tau) d \tau} .
\end{gathered}
$$

Sufficiency. We set

$$
\begin{aligned}
P_{1}(t) & =P_{-}(t), P_{2}(t) \\
& =Q_{+}(t), P_{3}(t) \\
& =P_{+}(t)-P_{-}(t), \\
& \text { for } t \in \mathbb{R} .
\end{aligned}
$$

First, we observe that

$$
\begin{gathered}
Q_{+}(t) Q_{-}(t)=Q_{-}(t) Q_{+}(t)=Q_{+}(t), \quad t \in \mathbb{R}, \\
P_{+}(t)-P_{-}(t)=P_{+}(t) Q_{-}(t)=Q_{-}(t) P_{+}(t), \quad t \in \mathbb{R} .
\end{gathered}
$$

The restriction $U(t, s)_{\mid \mathscr{R}\left(P_{2}(s)\right)}: \mathscr{R}\left(P_{2}(s)\right) \rightarrow \mathscr{R}\left(P_{2}(t)\right)$ is an isomorphism for all $t \geq s$ and, by relation (25), we have

$$
\begin{aligned}
& U_{P_{2}}(s, t) P_{2}(t) \\
& = \begin{cases}U_{Q_{+}}(s, t) Q_{+}(t) & \text { if } t \geq s \geq 0 \\
Q_{+}(s) U_{Q_{-}}(s, t) Q_{-}(t) Q_{+}(t) & \text { if } 0 \geq t \geq s \\
Q_{+}(s) U_{Q_{-}}(s, 0) Q_{-}(0) U_{Q_{+}}(0, t) Q_{+}(t) & \text { if } t \geq 0 \geq s .\end{cases}
\end{aligned}
$$

Also, the restriction $U(t, s)_{\mid \mathscr{R}\left(P_{3}(s)\right)}: \mathscr{R}\left(P_{3}(s)\right) \rightarrow \mathscr{R}\left(P_{3}(t)\right)$ is an isomorphism for all $(t, s) \in \Delta$ with $0 \geq t \geq s$ and, by relation (26), we deduce that

$$
U_{P_{3}}(s, t) P_{3}(t)=P_{+}(s) U_{Q_{-}}(s, t) Q_{-}(t) P_{+}(t) .
$$

We now verify the inequalities in Definition 6, considering three cases.

(1) For $t \geq s \geq 0$, we have

$$
\left\|U(t, s) P_{1}(s)\right\|=\left\|U(t, s) P_{+}(s) P_{-}(s)\right\|
$$

$$
\leq M_{-} K e^{-\int_{s}^{t} u(\tau) d \tau},
$$

$$
\begin{aligned}
\left\|U_{P_{2}}(s, t) P_{2}(t)\right\| & =\left\|U_{Q_{+}}(s, t) Q_{+}(t)\right\| \\
& \leq K e^{-\int_{s}^{t} u(\tau) d \tau},
\end{aligned}
$$

$$
\begin{aligned}
\left\|U(t, s) P_{3}(s)\right\| & =\left\|U(t, s) P_{+}(s) Q_{-}(s)\right\| \\
& \leq\left(1+M_{-}\right) K e^{-\int_{s}^{t} u(\tau) d \tau} .
\end{aligned}
$$

(2) If $0 \geq t \geq s$, then we obtain

$$
\begin{gathered}
\left\|U(t, s) P_{1}(s)\right\|=\left\|U(t, s) P_{-}(s)\right\| \leq K e^{-\int_{s}^{t} u(\tau) d \tau}, \\
\left\|U_{P_{2}}(s, t) P_{2}(t)\right\| \leq\left\|Q_{+}(s)\right\|\left\|U_{Q_{-}}(s, t) Q_{-}(t)\right\| \\
\times\left\|Q_{+}(t)\right\| \leq\left(1+M_{+}\right)^{2} K e^{-\int_{s}^{t} u(\tau) d \tau}, \\
\left\|U_{P_{3}}(s, t) P_{3}(t)\right\| \leq \\
\quad\left\|P_{+}(s)\right\|\left\|U_{Q_{-}}(s, t) Q_{-}(t)\right\| \\
\times\left\|P_{+}(t)\right\| \leq M_{+}^{2} K e^{-\int_{s}^{t} u(\tau) d \tau .}
\end{gathered}
$$


(3) For $t \geq 0 \geq s$, using the evolution property $\left(e_{2}\right)$, we get

$$
\begin{aligned}
\left\|U(t, s) P_{1}(s)\right\|= & \left\|U(t, s) P_{-}(s)\right\| \\
= & \left\|U(t, 0) P_{+}(0) U(0, s) P_{-}(s)\right\| \\
\leq & \left\|U(t, 0) P_{+}(0)\right\|\left\|U(0, s) P_{-}(s)\right\| \\
\leq & K e^{-\int_{0}^{t} u(\tau) d \tau} K e^{-\int_{s}^{0} u(\tau) d \tau} \\
= & K^{2} e^{-\int_{s}^{t} u(\tau) d \tau}, \\
\left\|U_{P_{2}}(s, t) P_{2}(t)\right\| \leq & \left\|Q_{+}(s)\right\| \\
& \times\left\|U_{Q_{-}}(s, 0) Q_{-}(0)\right\|\left\|U_{Q_{+}}(0, t) Q_{+}(t)\right\| \\
\leq & \left(1+M_{+}\right) K e^{-\int_{s}^{0} u(\tau) d \tau} K e^{-\int_{0}^{t} u(\tau) d \tau} \\
= & \left(1+M_{+}\right) K^{2} e^{-\int_{s}^{t} u(\tau) d \tau} .
\end{aligned}
$$

These complete the proof of the theorem.

Remark 12. Note that, in comparison to Lemma 1.2 in [11], we assume that $P_{+}(t)$ is bounded for $t \leq 0$ and $P_{-}(t)$ is bounded for $t \geq 0$. In our opinion, these conditions must also be added in the particular case of evolution operators generated by differential equations with bounded coefficients. This is motivated by the fact that the proof of the above mentioned lemma seems to not be quite accurate. More precisely, the first computation in (ii) $\Rightarrow$ (iii) holds only for $t \geq 0 \geq s$, and not for all $t \geq s$. Indeed, using the notations from [11] and taking, for example, the case $t \geq s \geq 0$, we have

$$
\begin{aligned}
\left\|X(t) P_{1} X^{-1}(s)\right\| & =\left\|X(t) P_{+} P_{-} X^{-1}(s)\right\| \\
& \leq\left\|X(t) P_{+} X^{-1}(s)\right\|\left\|X(s) P_{-} X^{-1}(s)\right\| \\
& \leq L e^{-\alpha(t-s)}\left\|X(s) P_{-} X^{-1}(s)\right\| .
\end{aligned}
$$

Thus, in order to prove (iii), one must add the assumption that there exists a constant $M_{-} \geq 1$ such that

$$
\left\|X(t) P_{-} X^{-1}(t)\right\| \leq M_{-}, \quad \forall t \geq 0 .
$$

In our case, this is equivalent to

$$
\sup _{t \geq 0}\left\|P_{-}(t)\right\| \leq M_{-}<\infty .
$$

The projection valued function $P_{-}$is defined on the whole real line. Because the evolution operator $U$ has a generalized exponential dichotomy with $P_{-}$only on the left half-line, we have that $P_{-}(t)$ is bounded for $t \leq 0$. Still, this does not give any information about the boundedness for $t>0$ (the same comment applies to $P_{+}$).
Theorem 13. An evolution operator $U: \Delta \rightarrow \mathscr{B}(X)$ has a generalized $\ell$-exponential trichotomy if and only if there exist two projection valued functions $P, Q: \mathbb{R} \rightarrow \mathscr{B}(X)$, which are invariant for $U$, and there exist a constant $K \geq 1$ and a function $u \in \mathcal{U}$ such that the following properties hold:

(1) $P(t)+Q(t)-P(t) Q(t)=I$ and $P(t) Q(t)=Q(t) P(t)$, for all $t \in \mathbb{R}$;

(2) $\sup _{t \leq 0}\|P(t)\| \leq M_{1}$ and $\sup _{t \geq 0}\|Q(t)\| \leq M_{2}$;

(3) the restriction of $U(t, s)$ on $\mathscr{R}(I-P(s))$ into $\mathscr{R}(I-P(t))$ is an isomorphism for all $(t, s) \in \Delta$ with $t \geq 0$;

(4) the restriction of $U(t, s)$ on $\mathscr{R}(Q(s))$ into $\mathscr{R}(Q(t))$ is an isomorphism for all $(t, s) \in \Delta_{-}$;

(5) $\|U(t, s) P(s)\| \leq K e^{-\int_{s}^{t} u(\tau) d \tau}$, for $(t, s) \in \Delta_{+}$;

(6) $\left\|U_{I-P}(s, t)(I-P(t))\right\| \leq K e^{-\int_{s}^{t} u(\tau) d \tau}$, for $(t, s) \in \Delta$ with $t \geq 0$;

(7) $\|U(t, s)(I-Q(s))\| \leq K e^{-\int_{s}^{t} u(\tau) d \tau}$, for $(t, s) \in \Delta$ with $s \leq 0$

(8) $\left\|U_{Q}(s, t) Q(t)\right\| \leq K e^{-\int_{s}^{t} u(\tau) d \tau}$, for $(t, s) \in \Delta_{-}$.

Proof. Necessity. We consider

$$
P(t)=P_{1}(t)+P_{3}(t), \quad Q(t)=P_{2}(t)+P_{3}(t),
$$

for $t \in \mathbb{R}$. It is easy to see that $P(t) Q(t)=Q(t) P(t)=P_{3}(t)$. Hence, $P(t)+Q(t)-P(t) Q(t)=I$. On the other hand, since $I-P(t)=P_{2}(t)$, we have that the restriction $U(t, s)_{\mid \mathscr{R}(I-P(s))}$ is an isomorphism from the range of $I-P(s)$ onto the range of $I-P(t)$ for all $(t, s) \in \Delta$ with $t \geq 0$ and

$$
U_{I-P}(s, t)=U_{P_{2}}(s, t) .
$$

By a similar argument as in the proof of Theorem 11, we obtain that $U(t, s)_{\mid \mathscr{R}(\mathrm{Q}(s))}$ is an isomorphism from the range of $Q(s)$ onto the range of $Q(t)$ for all $(t, s) \in \Delta_{-}$and

$$
U_{Q}(s, t) Q(t)=U_{P_{2}}(s, t) P_{2}(t)+U_{P_{3}}(s, t) P_{3}(t) .
$$

For $(t, s) \in \Delta_{+}$, we have

$$
\begin{aligned}
\|U(t, s) P(s)\| & \leq\left\|U(t, s) P_{1}(s)\right\|+\left\|U(t, s) P_{3}(s)\right\| \\
& \leq 2 K e^{-\int_{s}^{t} u(\tau) d \tau},
\end{aligned}
$$

and for $(t, s) \in \Delta_{-}$, we get

$$
\begin{aligned}
\left\|U_{Q}(s, t) Q(t)\right\| & \leq\left\|U_{P_{2}}(s, t) P_{2}(t)\right\|+\left\|U_{P_{3}}(s, t) P_{3}(t)\right\| \\
& \leq 2 K e^{-\int_{s}^{t} u(\tau) d \tau} .
\end{aligned}
$$

If $(t, s) \in \Delta$ with $t \geq 0$, then it follows that

$$
\begin{aligned}
\left\|U_{I-P}(s, t)(I-P(t))\right\| & =\left\|U_{P_{2}}(s, t) P_{2}(t)\right\| \\
& \leq K e^{-\int_{s}^{t} u(\tau) d \tau},
\end{aligned}
$$


and if $(t, s) \in \Delta$ with $s \leq 0$, then we have

$$
\|U(t, s)(I-Q(s))\|=\left\|U(t, s) P_{1}(s)\right\| \leq K e^{-\int_{s}^{t} u(\tau) d \tau} .
$$

Sufficiency. We set

$$
\begin{aligned}
& P_{1}(t)=I-Q(t), \\
& P_{2}(t)=I-P(t), \\
& P_{3}(t)=P(t) Q(t),
\end{aligned}
$$

for $t \in \mathbb{R}$. We observe that $I-P(t)=Q(t)(I-P(t))=(I-$ $P(t)) Q(t)$ for all $t \in \mathbb{R}$. Now, it is easy to see that the restriction of $U(t, s)$ on $\mathscr{R}\left(P_{2}(s)\right)$ into $\mathscr{R}\left(P_{2}(t)\right)$ is an isomorphism for all $t \geq s$ and

$U_{P_{2}}(s, t) P_{2}(t)$

$= \begin{cases}U_{I-P}(s, t)(I-P(t)) & \text { for } t \geq s \text { with } t \geq 0 \\ (I-P(s)) U_{Q}(s, t) Q(t)(I-P(t)) & \text { for } 0 \geq t \geq s .\end{cases}$

Also, the restriction $U(t, s)_{\mid \mathscr{R}\left(P_{3}(s)\right)}: \mathscr{R}\left(P_{3}(s)\right) \rightarrow \mathscr{R}\left(P_{3}(t)\right)$ is an isomorphism for all $(t, s) \in \Delta_{-}$and

$$
U_{P_{3}}(s, t) P_{3}(t)=P(s) U_{Q}(s, t) Q(t) P(t) .
$$

Let $(t, s) \in \Delta$. If $t \geq s \geq 0$, then we have

$$
\begin{aligned}
\left\|U(t, s) P_{1}(s)\right\| & =\|U(t, s)(I-Q(s))\| \\
& =\|U(t, s) P(s)(I-Q(s))\| \\
& \leq\|U(t, s) P(s)\|\|I-Q(s)\| \\
& \leq\left(1+M_{2}\right) K e^{-\int_{s}^{t} u(\tau) d \tau}, \\
\left\|U_{P_{2}}(s, t) P_{2}(t)\right\| & =\left\|U_{I-P}(s, t)(I-P(t))\right\| \\
& \leq K e^{-\int_{s}^{t} u(\tau) d \tau}, \\
\left\|U(t, s) P_{3}(s)\right\| & \leq\|U(t, s) P(s)\|\|Q(s)\| \\
& \leq M_{2} K e^{-\int_{s}^{t} u(\tau) d \tau} .
\end{aligned}
$$

If $0 \geq t \geq s$, then it follows

$$
\begin{aligned}
\left\|U(t, s) P_{1}(s)\right\|= & \|U(t, s)(I-Q(s))\| \\
\leq & K e^{-\int_{s}^{t} u(\tau) d \tau}, \\
\left\|U_{P_{2}}(s, t) P_{2}(t)\right\| \leq & \|I-P(s)\| \\
& \times\left\|U_{Q}(s, t) Q(t)\right\|\|I-P(t)\| \\
\leq & \left(1+M_{1}\right)^{2} K e^{-\int_{s}^{t} u(\tau) d \tau}, \\
\left\|U_{P_{3}}(s, t) P_{3}(t)\right\| \leq & \|P(s)\|\left\|U_{Q}(s, t) Q(t)\right\|\|P(t)\| \\
\leq & M_{1}^{2} K e^{-\int_{s}^{t} u(\tau) d \tau} .
\end{aligned}
$$

Finally, if $t \geq 0 \geq s$, then we get

$$
\begin{gathered}
\left\|U(t, s) P_{1}(s)\right\|=\|U(t, s)(I-Q(s))\| \leq K e^{-\int_{s}^{t} u(\tau) d \tau}, \\
\left\|U_{P_{2}}(s, t) P_{2}(t)\right\|=\left\|U_{I-P}(s, t)(I-P(t))\right\| \leq K e^{-\int_{s}^{t} u(\tau) d \tau} .
\end{gathered}
$$

Therefore, $U$ has a generalized $\ell$-exponential trichotomy.

3.2. Generalized r-Exponential Trichotomy. Now we consider a concept of generalized exponential trichotomy which is dual in a certain sense to the one given in Definition 6.

Definition 14. We say that an evolution operator $U$ has a generalized r-exponential trichotomy if there exist three supplementary projection valued functions $P_{1}, P_{2}$, and $P_{3}$ and there exist a constant $K \geq 1$ and a function $u \in \mathscr{U}$ such that the following properties hold:

$\left(r_{1}\right) P_{1}$ is invariant for $U$;

$\left(r_{2}\right) P_{2}$ is compatible with $U$;

$\left(r_{3}\right) P_{3}$ is compatible on the right with $U$;

$\left(r_{4}\right)\left\|U(t, s) P_{1}(s)\right\| \leq K e^{-\int_{s}^{t} u(\tau) d \tau}$, for all $(t, s) \in \Delta ;$

$\left(r_{5}\right)\left\|U_{P_{2}}(s, t) P_{2}(t)\right\| \leq K e^{-\int_{s}^{t} u(\tau) d \tau}$, for all $(t, s) \in \Delta$;

$\left(r_{6}\right)\left\|U(t, s) P_{3}(s)\right\| \leq K e^{-\int_{s}^{t} u(\tau) d \tau}$, for all $(t, s) \in \Delta_{-} ;$

$\left(r_{7}\right)\left\|U_{P_{3}}(s, t) P_{3}(t)\right\| \leq K e^{-\int_{s}^{t} u(\tau) d \tau}$, for all $(t, s) \in \Delta_{+}$.

If there is a positive constant $a>0$ such that $u(t) \geq a$ for all $t \in \mathbb{R}$, then we say that $U$ has an $r$-exponential trichotomy.

The notion of $r$-exponential trichotomy was defined in [13] for reversible evolution operators.

Remark 15. If $U$ has a generalized $r$-exponential trichotomy with projection valued functions $P_{1}, P_{2}$, and $P_{3}$, then we have that

$$
\sup _{t \in \mathbb{R}}\left\|P_{i}(t)\right\|<\infty, \quad \text { for } i \in\{1,2,3\} .
$$

Remark 16. If in Definition 6 we consider $P_{3}(t)=0$ for all $t \in \mathbb{R}$, then we obtain the concept of generalized exponential dichotomy (on the real line).

Example 17. The evolution operator $U: \Delta \rightarrow \mathscr{B}\left(\mathbb{R}^{3}\right)$ defined by

$$
\begin{aligned}
& U(t, s)\left(x_{1}, x_{2}, x_{3}\right) \\
& \quad=\left(e^{-\int_{s}^{t} u(\tau) d \tau} x_{1}, e^{\int_{s}^{t} u(\tau) d \tau} x_{2}, e^{\int_{s}^{t} \operatorname{sign}(\tau) u(\tau) d \tau} x_{3}\right)
\end{aligned}
$$

has a generalized $r$-exponential trichotomy with canonical projections for each $u \in \mathcal{U}$.

Proceeding in a similar manner to that in Theorems 11 and 13 , we get the following. 
Theorem 18. Let $U: \Delta \rightarrow \mathscr{B}(X)$ be an evolution operator. The following statements are equivalent:

(i) $U$ has a generalized $r$-exponential trichotomy;

(ii) There exist two projection valued functions $P_{+}$and $P_{-}$ invariant for $U$ and there exist a constant $K \geq 1$ and a function $u \in \mathcal{U}$ such that the following properties hold:

(1) $P_{+}(t) P_{-}(t)=P_{-}(t) P_{+}(t)=P_{+}(t)$, for all $t \in \mathbb{R}$;

(2) $\sup _{t \leq 0}\left\|P_{+}(t)\right\| \leq M_{+}$and $\sup _{t \geq 0}\left\|P_{-}(t)\right\| \leq M_{-}$;

(3) The restriction of $U(t, s)$ on $\mathscr{R}\left(Q_{+}(s)\right)$ into $\mathscr{R}\left(Q_{+}(t)\right)$ is an isomorphism for all $(t, s) \in \Delta_{+}$;

(4) The restriction of $U(t, s)$ on $\mathscr{R}\left(Q_{-}(s)\right)$ into $\mathscr{R}\left(Q_{-}(t)\right)$ is an isomorphism for all $(t, s) \in \Delta_{-}$;

(5) $\left\|U(t, s) P_{+}(s)\right\| \leq K e^{-\int_{s}^{t} u(\tau) d \tau}$, for all $(t, s) \in \Delta_{+}$;

(6) $\left\|U_{Q_{+}}(s, t) Q_{+}(t)\right\| \leq K e^{-\int_{s}^{t} u(\tau) d \tau}$, for all $(t, s) \in \Delta_{+}$;

(7) $\left\|U(t, s) P_{-}(s)\right\| \leq K e^{-\int_{s}^{t} u(\tau) d \tau}$, for all $(t, s) \in \Delta_{-}$;

(8) $\left\|U_{Q_{-}}(s, t) Q_{-}(t)\right\| \leq K e^{-\int_{s}^{t} u(\tau) d \tau}$, for all $(t, s) \in \Delta_{-}$;

where $Q_{+}(t)=I-P_{+}(t)$ and $Q_{-}(t)=I-P_{-}(t), t \in \mathbb{R}$;

(iii) There exist two projection valued functions $P$ and $Q$ invariant for $U$ and there exist a constant $K \geq 1$ and a function $u \in \mathscr{U}$ such that

(1) $P(t) Q(t)=Q(t) P(t)=0$, for all $t \in \mathbb{R}$;

(2) $\sup _{t \leq 0}\|P(t)\| \leq M_{1}$ and $\sup _{t \geq 0}\|Q(t)\| \leq M_{2}$;

(3) The restriction of $U(t, s)$ on $\mathscr{R}(I-P(s))$ into $\mathscr{R}(I-P(t))$ is an isomorphism for all $(t, s) \in \Delta_{+}$;

(4) The restriction of $U(t, s)$ on $\mathscr{R}(Q(s))$ into $\mathscr{R}(Q(t))$ is an isomorphism for all $(t, s) \in \Delta$ with $s \leq 0$;

(5) $\|U(t, s) P(s)\| \leq K e^{-\int_{s}^{t} u(\tau) d \tau}$, for $(t, s) \in \Delta$ with $t \geq 0$;

(6) $\left\|U_{I-P}(s, t)(I-P(t))\right\| \leq K e^{-\int_{s}^{t} u(\tau) d \tau}$, for $(t, s) \in \Delta_{+}$;

(7) $\|U(t, s)(I-Q(s))\| \leq K e^{-\int_{s}^{t} u(\tau) d \tau}$, for $(t, s) \in \Delta_{-}$;

(8) $\left\|U_{Q}(s, t) Q(t)\right\| \leq K e^{-\int_{s}^{t} u(\tau) d \tau}$, for $(t, s) \in \Delta$ with $s \leq 0$.

Let us notice that the exponential trichotomy considered in Definition 14 seems to be closer to exponential dichotomy on the real line than the other trichotomies, since in the case of linear differential equations it implies exponential dichotomy on both half lines and (3) has no nontrivial bounded solution (see Lemma 1 in [20]).

Remark 19. As a consequence of Lemma 2.2 from [11], the differential equation

$$
\dot{x}(t)=A(t) x(t), \quad t \in \mathbb{R},
$$

where $x(t) \in \mathbb{C}^{n}$ and $A(t)$ is a bounded and continuous $n \times n$ matrix on the whole real line, that is, $\sup _{t \in \mathbb{R}}\|A(t)\|<\infty$, has an $\ell$-exponential trichotomy if and only if its adjoint equation

$$
\dot{y}(t)=-A^{*}(t) y(t), \quad t \in \mathbb{R},
$$

has an $r$-exponential trichotomy, where $A^{*}(t)$ is the adjoint matrix of $A(t)$. When assuming that (50) is defined on a
Hilbert space $\mathscr{H}$, this result still holds. In fact, it remains valid for any reversible evolution operator on reflexive Banach spaces, in a certain sense, which we explain below.

If $U: \mathbb{R}^{2} \rightarrow \mathscr{B}(X)$ is a reversible evolution operator on a Banach space $X$, then we put

$$
V(t, s)=U(s, t)^{*}, \quad \text { for } t, s \in \mathbb{R} .
$$

Notice that $V: \mathbb{R}^{2} \rightarrow \mathscr{B}(X)$ is also a reversible evolution operator on the dual space $X^{*}$ of $X$ (see, e.g., [21]). Let us denote the elements of the dual space $X^{*}$ by $x^{*}$ and denote $\left\langle x, x^{*}\right\rangle=x^{*}(x)$. If $X^{* *}$ is the dual space of $X^{*}$, then there exists a continuous linear transformation $J: X \rightarrow X^{* *}$ defined by

$$
\left\langle x^{*}, J x\right\rangle=\left\langle x, x^{*}\right\rangle, \quad \forall x \in X \text { and } x^{*} \in X^{*} .
$$

As a consequence of the Hahn-Banach theorem, the operator $J$ is an isometry (i.e., $\|J x\|=\|x\|$, for every $x \in X$ ) and, hence, it is a one-to-one mapping.

Proposition 20. A reversible evolution operator $U$ on a reflexive Banach space $X$ has a generalized $\ell$-exponential trichotomy (generalized $r$-exponential trichotomy, respectively), if and only if its adjoint evolution operator $V$ has a generalized $r$ exponential trichotomy (generalized $\ell$-exponential trichotomy, respectively).

Proof. Necessity. It is easy to see that if $U$ has a generalized $\ell$-exponential trichotomy (a generalized $r$-exponential trichotomy) with projection valued functions $P_{+}(t)$ and $P_{-}(t)$ as in Theorem 11 (Theorem 18), then the adjoint evolution operator $V$ has a generalized $r$-exponential trichotomy (a generalized $\ell$-exponential trichotomy) with projections

$$
I-P_{+}^{*}(t) \text { and } I-P_{-}^{*}(t) .
$$

Sufficiency. We now assume that $V$ has a generalized $\ell$ exponential trichotomy with projection valued functions $P_{+}$ and $P_{-}$as in Theorem 11. For each $t \in \mathbb{R}$, we consider the following operators:

$$
\begin{array}{ll}
\widetilde{P}_{+}(t): X \longrightarrow X, & \widetilde{P}_{+}(t) x=J^{-1} P_{+}^{*}(t) J x, \\
\widetilde{P}_{-}(t): X \longrightarrow X, & \widetilde{P}_{-}(t) x=J^{-1} P_{-}^{*}(t) J x .
\end{array}
$$

We notice that $\widetilde{P}_{+}$and $\widetilde{P}_{-}$are projection valued functions on $X$. We first prove that they are invariant for $U$. We have

$$
\begin{aligned}
\left\langle\widetilde{P}_{+}(t) U(t, s) x, x^{*}\right\rangle \\
=\left\langle x^{*}, J \widetilde{P}_{+}(t) U(t, s) x\right\rangle=\left\langle x^{*}, P_{+}^{*}(t) J U(t, s) x\right\rangle \\
=\left\langle P_{+}(t) x^{*}, J U(t, s) x\right\rangle=\left\langle U(t, s) x, P_{+}(t) x^{*}\right\rangle
\end{aligned}
$$




$$
\begin{aligned}
& =\left\langle x, U(t, s)^{*} P_{+}(t) x^{*}\right\rangle=\left\langle x, P_{+}(s) U(t, s)^{*} x^{*}\right\rangle \\
& =\left\langle P_{+}(s) U(t, s)^{*} x^{*}, J x\right\rangle=\left\langle U(t, s)^{*} x^{*}, P_{+}^{*}(s) J x\right\rangle \\
& =\left\langle U(t, s)^{*} x^{*}, J \widetilde{P}_{+}(s) x\right\rangle=\left\langle\widetilde{P}_{+}(s) x, U(t, s)^{*} x^{*}\right\rangle \\
& =\left\langle U(t, s) \widetilde{P}_{+}(s) x, x^{*}\right\rangle,
\end{aligned}
$$

for all $x \in X$ and $x^{*} \in X^{*}$, and we similarly get that

$$
\begin{array}{r}
\left\langle\widetilde{P}_{-}(t) U(t, s) x, x^{*}\right\rangle=\left\langle U(t, s) \widetilde{P}_{-}(s) x, x^{*}\right\rangle, \\
x \in X, \quad x^{*} \in X^{*} .
\end{array}
$$

These imply

$$
\begin{aligned}
& \widetilde{P}_{+}(t) U(t, s)=U(t, s) \widetilde{P}_{+}(s), \\
& \widetilde{P}_{-}(t) U(t, s)=U(t, s) \widetilde{P}_{-}(s) .
\end{aligned}
$$

Hence, $\widetilde{P}_{+}$and $\widetilde{P}_{-}$are both invariant for $U$. We can easily get

$$
\widetilde{P}_{+}(t) \widetilde{P}_{-}(t)=\widetilde{P}_{-}(t) \widetilde{P}_{+}(t)=\widetilde{P}_{-}(t), \quad t \in \mathbb{R} .
$$

It is not difficult to prove that $U$ has a generalized $r$ exponential trichotomy with projections $I-\widetilde{P}_{+}(t)$ and $I-\widetilde{P}_{-}(t)$ in Theorem 18. Similarly, we can obtain that if the evolution operator $V$ has a generalized $r$-exponential trichotomy, then $U$ has a generalized $\ell$-exponential trichotomy.

Remark 21. The necessity from the previous proposition still holds in a general Banach space. At this point, we do not know whether the sufficiency is also valid.

\section{Acknowledgment}

The authors would like to thank the referees for carefully reading their paper and for their helpful suggestions and comments, which improved the quality of the paper.

\section{References}

[1] L. Jiang, "Generalized exponential dichotomy and global linearization," Journal of Mathematical Analysis and Applications, vol. 315, no. 2, pp. 474-490, 2006.

[2] N. Lupa and M. Megan, "Exponential dichotomies of evolutionoperators in Banach spaces," Monatshefte für Mathematik. In press.

[3] R. O. Mosincat, C. Preda, and P. Preda, "Dichotomies with no invariant unstable manifolds for autonomous equations," Journal of Function Spaces and Applications, vol. 2012, Article ID 527647, 23 pages, 2012.

[4] J. S. Muldowney, "Dichotomies and asymptotic behavior for linear differential systems," Transactions of the American Mathematical Society, vol. 283, pp. 465-484, 1984.

[5] R. J. Sacker and G. R. Sell, "Existence of dichotomies and invariant splittings for linear differential systems, III," Journal of Differential Equations, vol. 22, no. 2, pp. 497-522, 1976.
[6] M. Megan and C. Stoica, "On uniform exponential trichotomy of evolution operators in Banach spaces," Integral Equations and Operator Theory, vol. 60, no. 4, pp. 499-506, 2008.

[7] M. Megan and C. Stoica, "Equivalent definitions for uniform exponential trichotomy of evolution operators in Banach spaces," Hot Topics in Operator Theory, vol. 9, pp. 151-158, 2008.

[8] B. Sasu and A. L. Sasu, "Exponential trichotomy and padmissibility for evolution families on the real line," Mathematische Zeitschrift, vol. 253, no. 3, pp. 515-536, 2006.

[9] B. Sasu and A. L. Sasu, "Nonlinear criteria for the existence of the exponential trichotomy in infinite dimensional spaces," Nonlinear Analysis, Theory, Methods and Applications, vol. 74, no. 15, pp. 5097-5110, 2011.

[10] J. Zhang, "Lyapunov function and exponential trichotomy on time scales," Discrete Dynamics in Nature and Society, vol. 2011, Article ID 958381, 22 pages, 2011.

[11] S. Elaydi and O. Hajek, "Exponential trichotomy of differential systems," Journal of Mathematical Analysis and Applications, vol. 129, no. 2, pp. 362-374, 1988.

[12] L. H. Popescu, N. Lupa, and M. Megan, "Exponential trichotomy on Banach spaces," in Proceedings of the Seminar of Mathematical Analysis and Applications in Control Theory, University of Timişoara, 2010.

[13] L. H. Popescu and T. Vesselenyi, "Trichotomy and topological equivalence for evolution families," Bulletin of the Belgian Mathematical Society, vol. 18, no. 4, pp. 679-694, 2011.

[14] S. Elaydi and O. Hajek, "Exponential dichotomy of nonlinear systems of ordinary differential equations," Differential Integral Equations, vol. 3, pp. 1201-1224, 1990.

[15] J. Hong, R. Obaya, and A. S. Gil, "Exponential trichotomy and a class of ergodic solutions of differential equations with ergodic perturbations," Applied Mathematics Letters, vol. 12, no. 1, pp. 7-13, 1999.

[16] R. Nagel and G. Nickel, "Wellposedness for nonautonomous abstract Cauchy problems," in Progress in Nonlinear Differential Equations and Their Applications, vol. 50, pp. 279-293, 2002.

[17] P. Acquistapace, "Evolution operators and strong solutions of abstract linear parabolic equations," Differential Integral Equations, vol. 1, pp. 433-457, 1988.

[18] W. A. Coppel, Dichotomies in Stability Theory, vol. 629 of Lecture Notes in Mathematics, Springer, Berlin, Germany, 1978.

[19] C. Pötzsche, "Nonautonomous bifurcation of bounded solutions II: a shovel-bifurcation pattern," Discrete and Continuous Dynamical Systems, vol. 31, no. 3, pp. 941-973, 2011.

[20] K. J. Palmer, "Exponential dichotomy and expansivity," Annali di Matematica Pura ed Applicata, vol. 185, supplement 5, pp. S171-S185, 2006.

[21] H. M. Rodrigues and J. G. Ruas, "Evolution equations: dichotomies and the Fredholm alternative for bounded solutions," Journal of Differential Equations, vol. 119, no. 2, pp. 263283, 1995. 


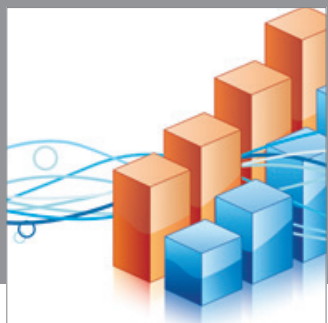

Advances in

Operations Research

mansans

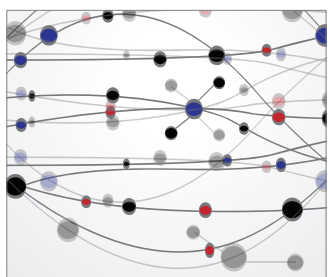

The Scientific World Journal
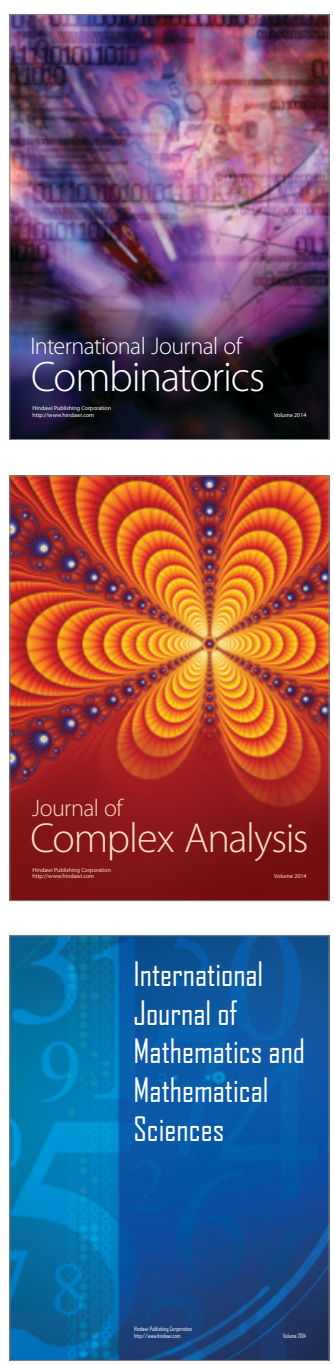
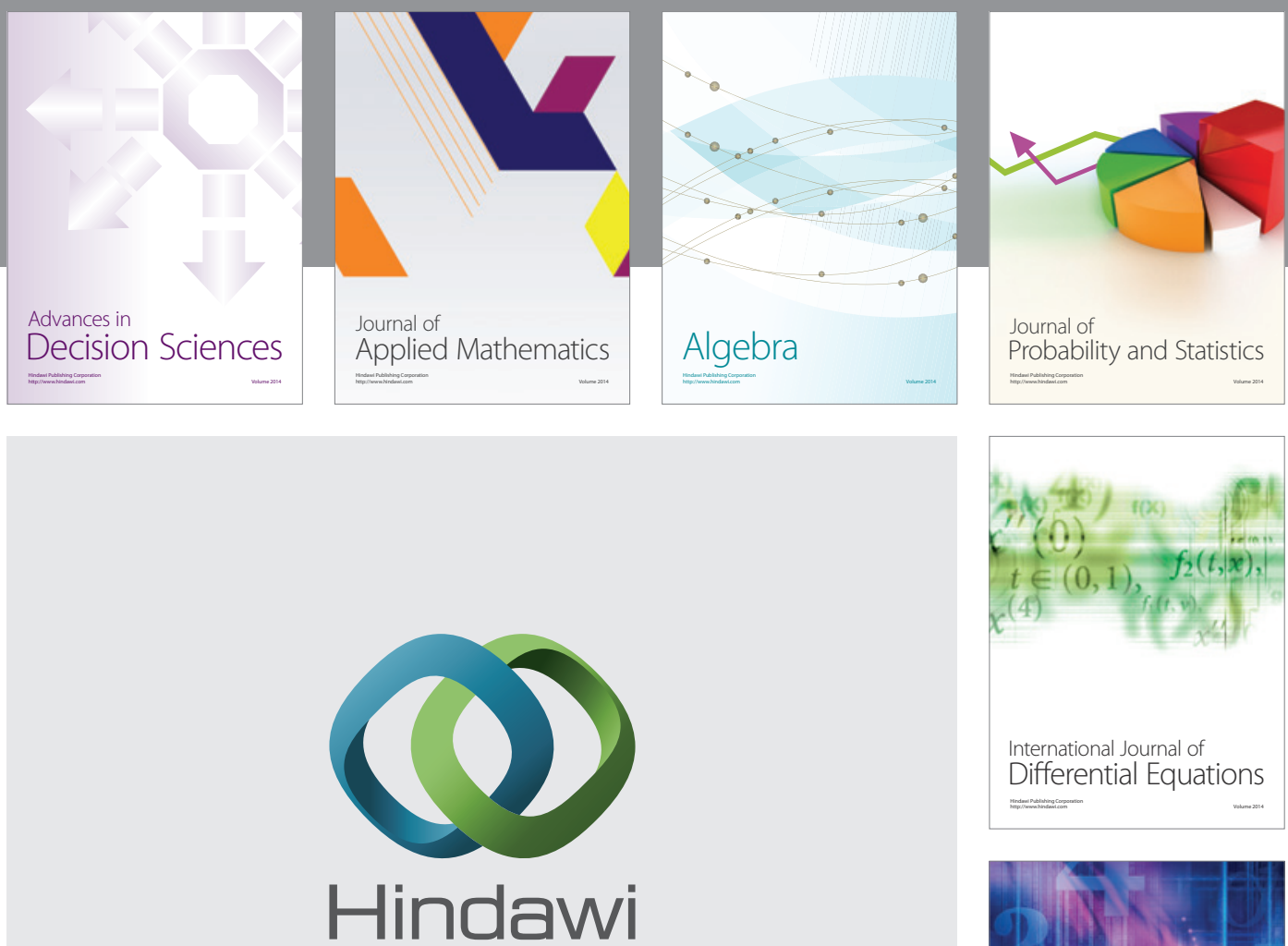

Submit your manuscripts at http://www.hindawi.com
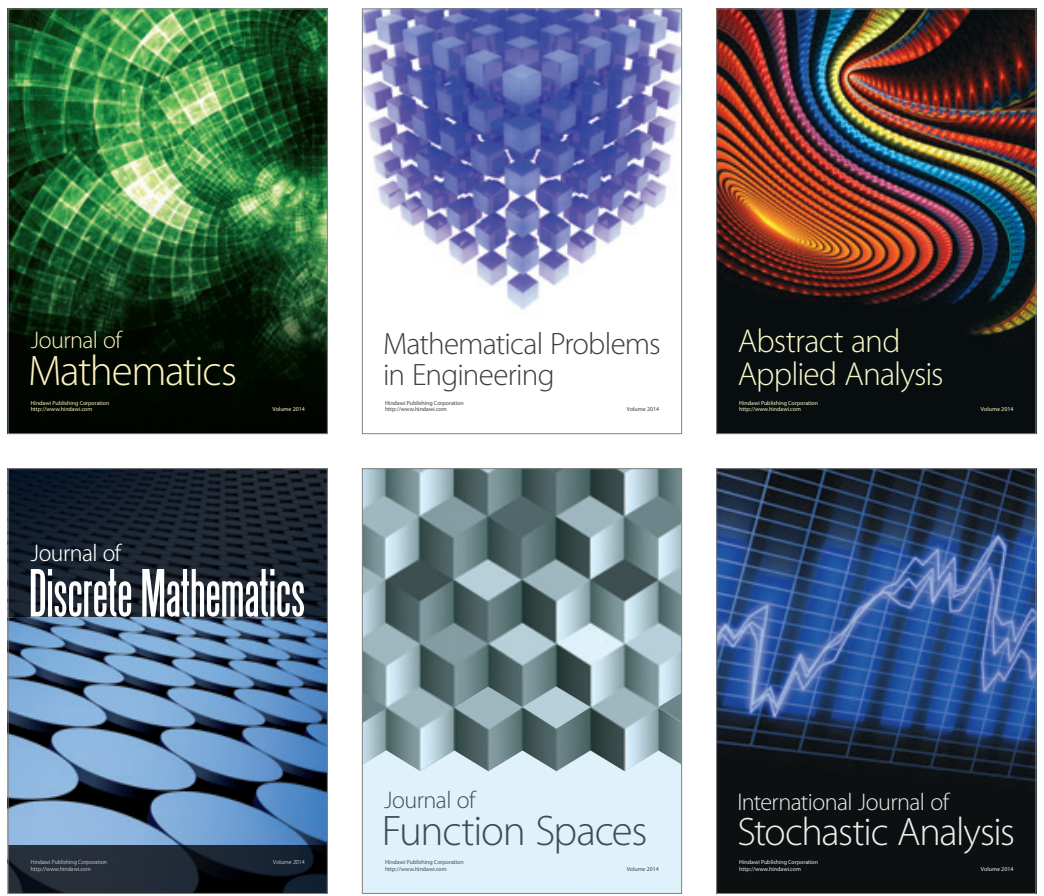

Journal of

Function Spaces

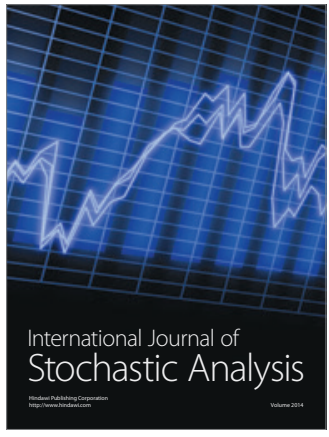

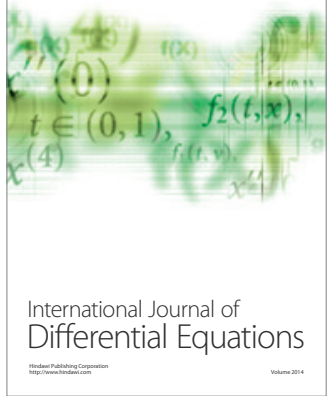
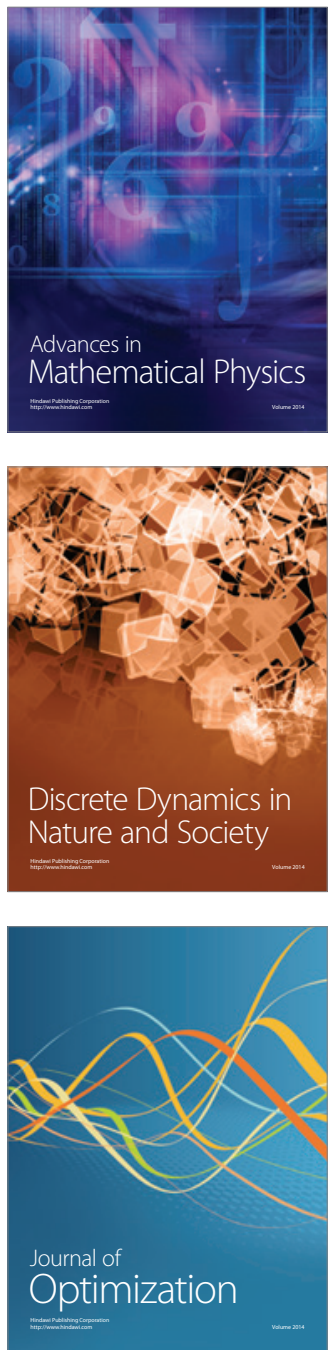